\title{
APPENDIX
}

\section{Women and Heart Disease: Risk Factor Assessment}




\section{NONMODIFIABLE}

A. Age: Heart disease is the leading cause of death for adult women; risks increase with age and after the menopause transition

B. Race or ethnicity: Black women have a higher rate of death than White women. Rates of diabetes and hypertension in Black women are higher than those in White women. Systemic racism is a significant contributing factor for heart disease among women of color.

C. Family history of the disease, especially myocardial infarction or stroke at age younger than 50 years old

D. Sex: Attenuating advantage of being a woman, especially premenopausally when women's risk is lower than men's

\section{MODIFIABLE}

\section{A. Lifestyle}

1. Exercise: Aerobic physical activity can reduce the risk of atherosclerotic cardiovascular disease (ASCVD). Adults should reduce overall sedentary behavior and engage in at least 150 minutes of accumulated moderate-intensity physical activity or 75 minutes of vigorous-intensity physical activity per week.

a. Moderate-intensity activity includes brisk walking (2.4-4 mph), biking (5-9 mph), dancing, active yoga, recreational swimming

b. Vigorous-intensity activity includes jogging, running, swimming laps, biking ( $\geq 10 \mathrm{mph}$ ), playing tennis, racquetball

2. Chronic diseases: Diabetes, obesity, and hypertension increase ASCVD. If present, these conditions should first be managed with lifestyle modification or pharmacologic therapies if lifestyle changes are ineffective.

3. Nutritional recommendations:

a. Replace saturated fat with monounsaturated and polyunsaturated fats

b. Overall fat intake between $20 \%$ and $35 \%$ of all calories $(20 \%$ is best)

c. Avoid trans fats

d. Less than $2,000 \mathrm{mg}$ day of sodium $(<1,500 \mathrm{mg}$ per day with hypertension)

e. Maintain body mass index (BMI) between 18.5 and $24.9 \mathrm{~kg} / \mathrm{m}^{2}$

f. Diet that emphasizes the intake of fruits, vegetables, legumes, fish, lean protein, nuts, and whole grains

g. Minimize intake of animal fats, red meat, processed foods, sugar, refined, simple carbohydrates, and sugary and sweetened drinks

h. Reduce alcohol intake to $\leq 1$ drinks per day

4. Cigarette smoking (including use of e-cigarettes)

a. Assess all women for tobacco use at every visit for the risk of adverse cardiovascular and thrombotic events

b. Advise all women of the health risks of tobacco and encourage quitting 
c. Assess readiness to quit and offer behavioral interventions and/ or pharmacotherapy to increase success with tobacco cessation

d. Encourage avoidance of secondhand smoke when possible

e. Caution regarding the risks of e-cigarettes

f. Smoking and use of combination hormonal contraception increases the risk of adverse cardiovascular and thrombotic events

B. Internal and external environmental factors

a. Management of comorbid health conditions

b. Psychosocial concerns: chronic stress contributes to comorbid health conditions. Encourage women to manage stress in their lives

c. Access to health care: Regular, preventive health care and age-related screening tests are an important component of cardiac risk reduction.

d. Encourage all women to explore options for health insurance.

e. Socioeconomic status: Inverse association with morbidity and mortality

\section{Resources}

www.ahajournals.org/doi/10.1161/CIR.0000000000000678

www.heart.org/en/health-topics/consumer-healthcare/what-is-cardiovasculardisease/heart-health-risk-assessments-from-the-american-heart-association 